\title{
Expression and significance of TIMP-3, PACAP and VIP in vaginal wall tissues of patients with stress urinary incontinence
}

\author{
BO FAN, XIAOHUA JIN, YI SHI, HAILIANG ZHU, WENJUN ZHOU, WENJIAN TU and LI DING \\ Department of Urology, Changshu No. 1 People's Hospital Affiliated of \\ Soochow University, Changshu, Jiangsu 215500, P.R. China
}

Received July 8, 2016; Accepted December 19, 2016

DOI: 10.3892/etm.2016.3988

\begin{abstract}
The objective of the present study was to investigate whether tissue inhibitor of metalloproteinase-3 (TIMP-3), pituitary adenylate cyclase-activating polypeptide (PACAP), and vasoactive intestinal peptide (VIP) participate in the occurrence of female stress urinary incontinence (SUI) by measuring the expression levels of TIMP-3,PACAP, and VIP in the vaginal wall and analyzing their correlation to understand the pathogenesis of female SUI. Forty female patients who were admitted to our hospital for tension-free obturator tape surgery for treatment of SUI from April, 2012 to December, 2015 were selected as the study group. Forty patients who underwent vaginal or total abdominal hysterectomy for treatment of non-estrogen-related diseases during the same period were selected as the control group. Tissue samples from the anterior vaginal wall, located at twelve o'clock, were taken from both groups. The expression levels of TIMP-3, PACAP and VIP were detected by immunohistochemistry, and the correlation of integral optical density (IOD) among expressions of TIMP-3, PACAP, and VIP was investigated. The expression of TIMP-3 in vaginal wall tissues of the study group was lower than that of the control group $(\mathrm{P}<0.05)$. The expression of PACAP and VIP in vaginal tissues of the study group were lower than those of the control group $(\mathrm{P}<0.05)$. In the study group, the IOD of PACAP expression was significantly and positively correlated with that of VIP $(\mathrm{r}=0.873, \mathrm{P}<0.05)$, the IOD of PACAP expression was significantly and positively correlated with that of TIMP-3 $(r=0.802$, $\mathrm{P}<0.05)$, and the IOD of VIP expression was significantly and positively correlated with that of TIMP-3 $(r=0.716, \mathrm{P}<0.05)$. In conclusion, TIMP-3, PACAP and VIP jointly participate in the occurrence of female SUI. Increasing the expression of TIMP-3, PACAP, and VIP, repairing neurons, and enhancing
\end{abstract}

Correspondence to: Dr Bo Fan, Department of Urology, Changshu No. 1 People's Hospital Affiliated of Soochow University, 1 Shuyuan Street, Changshu, Jiangsu 215500, P.R. China

E-mail: fan_bo_111@163.com

Key words: stress urinary incontinence, TIMP-3, PACAP, VIP, vaginal wall tissue the elasticity of vaginal wall tissues may become a new way to treat female SUI.

\section{Introduction}

Stress urinary incontinence (SUI) is a relatively common gynecological disease. The main clinical manifestations are increases in abdominal pressure, and urine leaks which patients are unaware of $(1,2)$. Previous studies have indicated that the factors that can affect female SUI include pelvic organ prolapse, age, procreation, and genetic factors, but the specific pathogenesis of this disease is not clear (3-5). Tissue inhibitor of metalloproteinase-3 (TIMP-3) plays an important role in the development of a variety of human tissues. It not only stimulates cell proliferation, but also induces apoptosis. Pituitary adenylate cyclase-activating polypeptide (PACAP) and vasoactive intestinal peptide (VIP), expressed by different tissues and organs in the body, have similar functions and play important roles in angiokinesis and pelvic floor muscle activity. In the present study, we aimed to investigate whether TIMP-3, PACAP, and VIP participate in the occurrence of female SUI, to understand the pathogenesis of the disease.

\section{Patients and methods}

Patients. Forty female patients, who were admitted to our hospital for tension-free obturator tape surgery for SUI from April, 2012 to December, 2015, were selected as the study group. Ages ranged from 46 to 56 years, with average age of $44.7 \pm 1.5$ years. The enrolled patients who met the diagnostic criteria of SUI were diagnosed by gynecological and urine dynamics examinations. Patients who had estrogen-related diseases such as genital and urinary tract infections, mental disorders, functional ovarian tumors, and patients with major organ diseases such as cardiopathy, were excluded. Simultaneously, 40 patients who underwent vaginal or total abdominal hysterectomy for treatment of non-estrogen-related diseases during the same period, were selected as the control group. Ages ranged from 45 to 55 years, with average age of $43.8 \pm 1.4$ years. Patients without symptoms of urinary incontinence were not enrolled in the control group. Patients with genital and urinary tract infections, liver cirrhosis, mental disorders, and acute inflammation were excluded. Patients in both groups did not take hormone drugs during the 3-month 
pre-operative period. The differences of general parameters between the two groups were not statistically significant $(\mathrm{P}>0.05)$.

Methods. Tissue samples of the anterior wall of the vagina, located at twelve o'clock, were taken from both groups of patients. The expression levels of TIMP-3, PACAP, and VIP were detected by immunohistochemistry and the correlation of the integral optical density (IOD) among expression of TIMP-3, PACAP, and VIP were investigated.

Preparation and staining of tissue sections. Tissue sample of the anterior vaginal wall, located at twelve o'clock, were collected from both groups of patients. Samples were washed in physiological saline and placed in $10 \%$ formaldehyde solution (mixed with neutral buffer) for fixation. Tissue samples were then embedded in paraffin and serial 3- $\mu \mathrm{m}$ sections were prepared.

The expression levels of TIMP-3, PACAP, and VIP of the tissue sections were detected by immunohistochemistry. PBS was used as the negative control instead of primary antibody. Diaminobenzidine was applied for coloration of the above sections, and hematoxylin was used to stain the nuclei (6).

Determination of results and image analysis. A light microscope was used to observe stained tissue sections. The cells with yellow-brown or yellow-sepia particle deposition in the cytoplasm or plasma membrane were considered positive cells. According to the positive cell rate and staining intensity of positive cells, the expression level was determined according to the following scoring system: Staining the same as the negative control was 0 points; staining showing pale yellow was 1 point; staining showing brown-yellow was 2 points; and staining showing sepia was 3 points. According to the proportion of positive cells, samples were divided as follows: $\leq 10 \%$ positive cells was 1 point; $11-50 \%$ was 2 points; $51-75 \%$ was 3 points; and $>75 \%$ was 4 points. The scores of the above two items were multiplied: 0-3 points was (-), 4-5 points was $(+), 6-7$ points was $(++),>8$ points was $(+++), \leq 3$ points was negative, and $>3$ points was positive.

Five typical high power fields were randomly selected, using a microscope image acquisition system (Hangzhou Huaxin Integrated Circuit Co., Ltd., Hangzhou, China), and the IOD of immunohistochemical-positive expression in each photo was automatically calculated by the professional image analysis software, Image-Pro Plus 6.1 (Media Cybernetics, Inc., Rockville, MD, USA).

Statistical analysis. SPSS 20.0 (IBM SPSS, Armonk, NY, USA) software was used for statistical analysis. Measurement data are presented as mean \pm standard deviation. The t-test was used for comparisons between the two groups. The Chi-square test was used for enumeration data. The correlation between indexes was analyzed by Pearson correlation analysis. $\mathrm{P}<0.05$ was considered to indicate a statistically significant difference.

\section{Results}

Expression of TIMP-3, PACAP, and VIP in vaginal tissue. The positive TIMP-3 expression product appeared as yellow

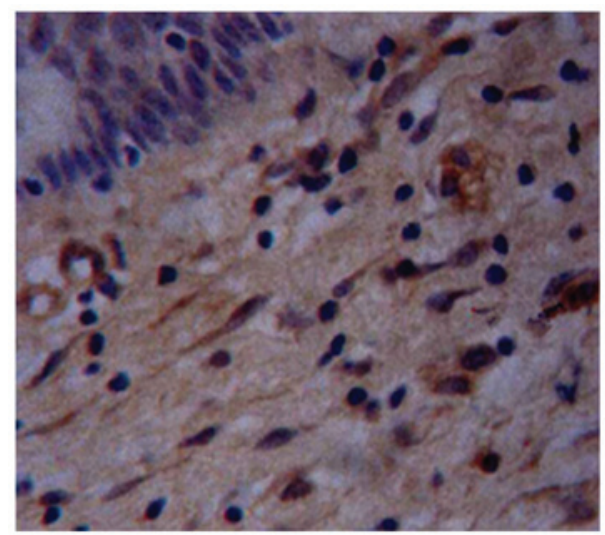

Figure 1. Tissue inhibitor of metalloproteinase-3 expression in study group (SP, $x 400)$.

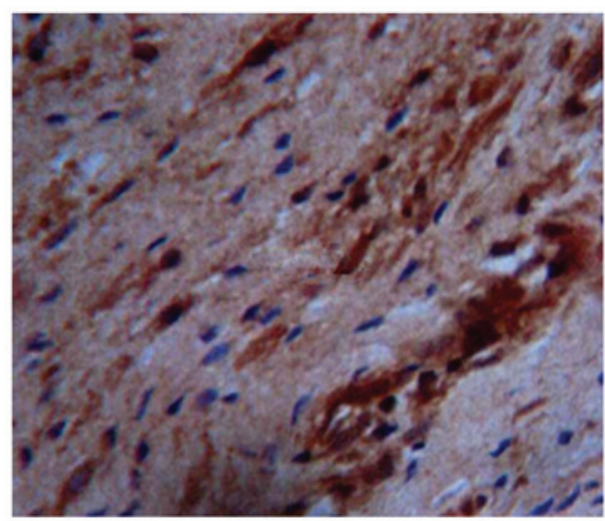

Figure 2. Tissue inhibitor of metalloproteinase-3 expression in control group (SP, x400).

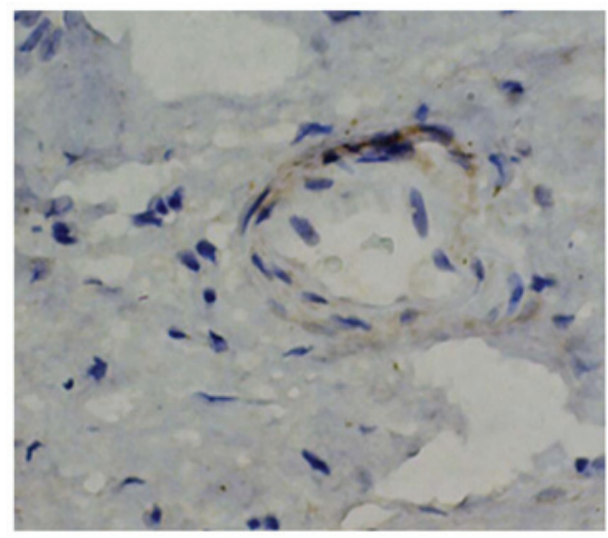

Figure 3. Pituitary adenylate cyclase-activating polypeptide expression in study group (SP, x400).

particles (Figs. 1 and 2). The PACAP and VIP positive expression products were brown-yellow particles (Figs. 2-6).

The positive rate of TIMP-3 expression was $27.5 \%$ in the study group, which was significantly lower than that of the control group $(47.5 \%)(\mathrm{P}<0.05)$ (Table I). The positive rate of PACAP expression was $25 \%$ in the study group, which was significantly lower than that of the control group $(42.5 \%)$ $(\mathrm{P}<0.05)$ (Table II). 
Table I. Expression of TIMP-3 in anterior vaginal wall tissue $(n=80)$.

\begin{tabular}{lccccccc}
\hline Groups & No. & + & ++ & +++ & Positive rate $(\%)$ & - & P-value \\
\hline Study & 40 & 3 & 3 & 5 & 27.5 & 29 & $<0.05$ \\
Control & 40 & 5 & 6 & 8 & 47.5 & 21 & $<0.05$ \\
\hline
\end{tabular}

TIMP-3, tissue inhibitor of metalloproteinase-3.

Table II. Expression of PACAP in anterior vaginal wall tissue $(n=80)$.

\begin{tabular}{lccccccc}
\hline Groups & No. & + & ++ & +++ & Positive rate $(\%)$ & - & P-value \\
\hline Study & 40 & 3 & 3 & 4 & 25.0 & 30 & $<0.05$ \\
Control & 40 & 4 & 6 & 7 & 42.5 & 23 & $<0.05$
\end{tabular}

PACAP, pituitary adenylate cyclase-activating polypeptide.

Table III. Expression of VIP in anterior vaginal wall tissue $(n=80)$.

\begin{tabular}{lcccccccc}
\hline Groups & No. & + & ++ & +++ & Positive rate $(\%)$ & - & P-value \\
\hline Study & 40 & 3 & 2 & 4 & 22.5 & 31 & $<0.05$ \\
Control & 40 & 4 & 7 & 7 & 45.0 & 22 & $<0.05$
\end{tabular}

VIP, vasoactive intestinal peptide.

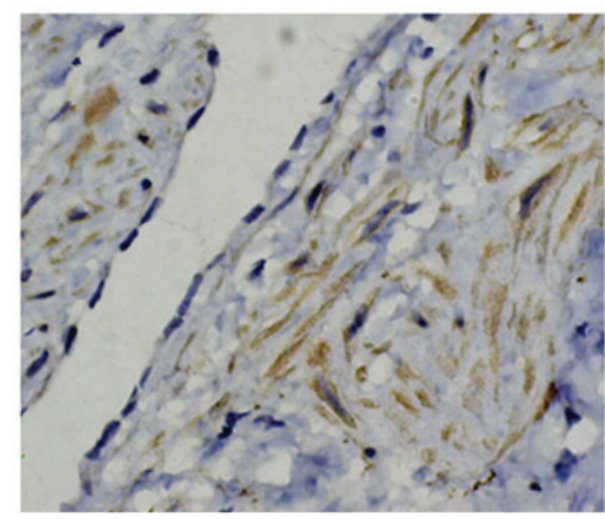

Figure 4. Pituitary adenylate cyclase-activating polypeptide expression in control group (SP, $\mathrm{x} 400)$.

The positive rate of VIP expression was $22.5 \%$ in the study group, which was significantly lower than that of control group $(45 \%)(\mathrm{P}<0.05)($ Table III).

Correlation analysis of PACAP and VIP expression in the study group. In the study group, the IOD of PACAP expression was significantly and positively correlated with that of VIP $(\mathrm{r}=0.873, \mathrm{P}<0.05)($ Fig. 7).

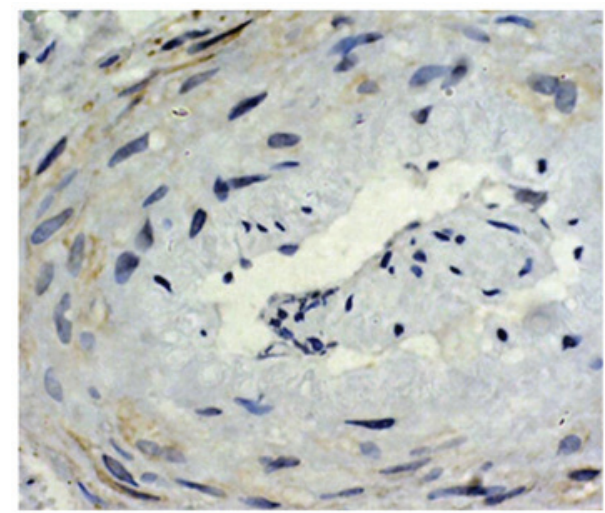

Figure 5. Vasoactive intestinal peptide expression in study group (SP, x400).

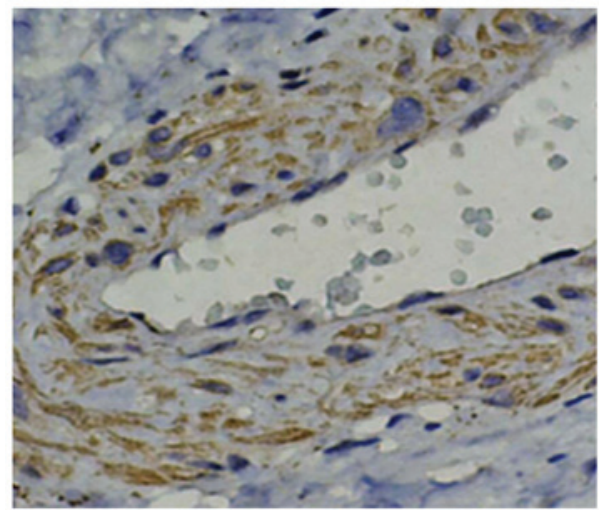

Figure 6. Vasoactive intestinal peptide expression in control group (SP, x400)

Correlation analysis of PACAP and TIMP-3 expression in the study group. In the study group, the IOD of PACAP expression was significantly and positively correlated with that of TIMP-3 $(\mathrm{r}=0.802, \mathrm{P}<0.05)($ Fig. 8).

Correlation analysis of VIP and TIMP-3 expression in the study group. In the study group, the IOD of VIP expression was significantly and positively correlated with that of TIMP-3 $(\mathrm{r}=0.716, \mathrm{P}<0.05)$ (Fig. 9).

\section{Discussion}

The urine of SUI patients leaks while the patient is unaware as a result of daily occurrences such as coughing and sneezing. Although this does not threaten the lives of patients, it can seriously affect quality of life. Previous studies have indicated that the etiology of SUI may be associated with birth injury, fecundity, the urethra, heredity, history of vaginal surgery, pelvic mass, and other factors (7-10). Thom et al (11) found through survey that there were differences in SUI of women of different ethnic backgrounds including Spanish, Asian American, Caucasian, and Black $(\mathrm{P}<0.05)$, showing that the occurrence of SUI was related to race and other factors.

With the rapid development of modern molecular biology, many scholars have investigated the mechanisms of SUI at the molecular level. Relevant study showed that the imbalance of calpain/calpastatin expression may be involved in the occurrence and development of SUI (12). TIMPs, which are proven 


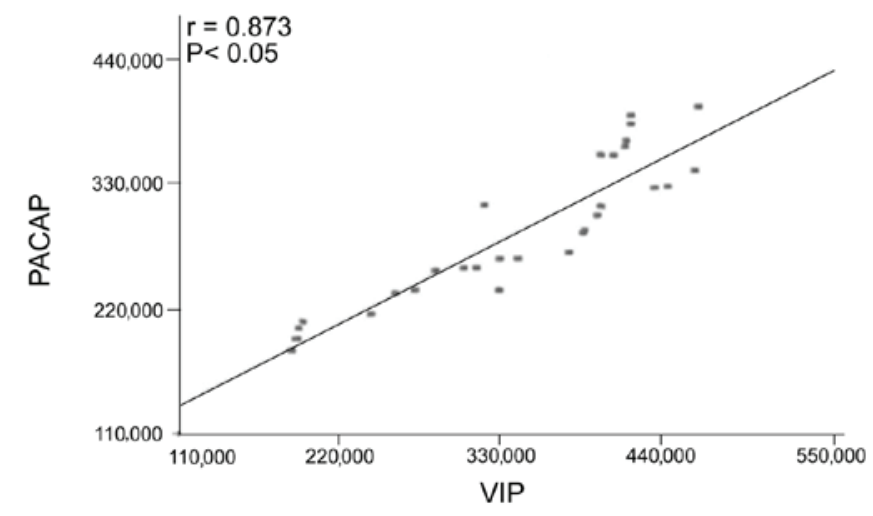

Figure 7. Correlation of pituitary adenylate cyclase-activating polypeptide (PACAP) and vasoactive intestinal peptide (VIP) expression in study group.

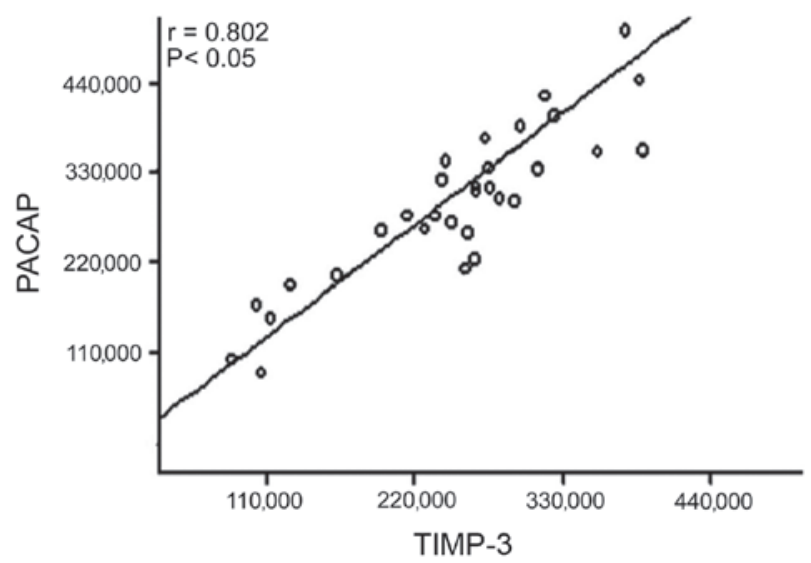

Figure 8. Correlation of pituitary adenylate cyclase-activating polypeptide (PACAP) and tissue inhibitor of metalloproteinase-3 (TIMP-3) expression in study group.

to inhibit matrix metalloproteinases (MMPs), have the ability to block the growth, invasion, and metastasis of tumors by inhibiting MMP function. TIMP-3 is an important member of the TIMP family, which only exists in the extracellular matrix (ECM), and combines with the ECM to form a non-soluble protein (13). TIMP-3 can inhibit MMP-induced degradation of ECM, while in contrast, it can induce apoptosis. Studies have shown that TIMP-3 expression was found in lung and prostate cancer tissues, but there are few studies reporting TIMP-3 expression in vaginal wall tissue of patients with SUI (14-16). In the present study, TIMP-3 expression was found in the vaginal wall tissues of patients of the study group and control group. The expression in the study group was significantly lower than that of the control group $(\mathrm{P}<0.05)$. This indicates that TIMP-3 expression is decreased in the vaginal wall tissue of patients with SUI, which may reduce the inhibition of MMPs, and may represent a significant mechanism to induce the occurrence of SUI.

VIP is a neurotransmitter in the human central and peripheral nervous systems, that positively mediates blood vessel expansion, non-vascular smooth muscle relaxation, pelvic blood flow, and vaginal lubrication $(17,18)$. There are abundant PACAP neural networks located in tissues near the blood vessels of the vaginal wall, where PACAP and VIP are co-expressed. Relevant studies have indicated that PACAP can actively participate in regulating and controlling human

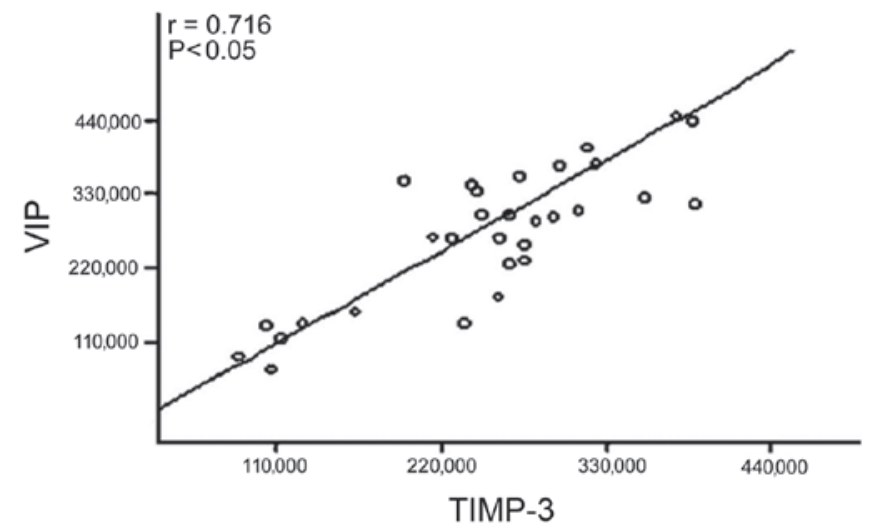

Figure 9. Correlation of vasoactive intestinal peptide (VIP) and tissue inhibitor of metalloproteinase-3 (TIMP-3) expression in study group.

vaginal blood flow and help relax vessels with VIP $(19,20)$. In the present study, expression of PACAP and VIP were detected in vaginal tissues from patients of the study and control groups. Expression in the study group was significantly lower than that of the control group $(\mathrm{P}<0.05)$. This indicated that SUI occurred because of decreased expression of PACAP and VIP, which may have resulted in neuronal damage.

IOD reflects the intensity of protein expression by positive cells. In the study group, the IOD of PACAP expression was significantly and positively correlated with that of VIP $(\mathrm{r}=0.873, \mathrm{P}<0.05)$, the IOD of PACAP expression was significantly and positively correlated with that of TIMP-3 ( $r=0.802$, $\mathrm{P}<0.05)$ and the IOD of VIP expression was significantly and positively correlated with that of TIMP-3 $(r=0.716, \mathrm{P}<0.05)$. All of the above results indicated that TIMP-3, PACAP, and VIP jointly participated in the occurrence of SUI.

In conclusion, TIMP-3, PACAP, and VIP jointly participate in the occurrence of female SUI. Increasing the expression of TIMP-3, PACAP, and VIP, repairing neurons, and enhancing the elasticity of vaginal wall tissues, may become potential ways to treat female SUI.

\section{References}

1. Anitua E, Troya M and Orive G: Plasma rich in growth factors promote gingival tissue regeneration by stimulating fibroblast proliferation and migration and by blocking transforming growth factor- $\beta 1$-induced myodifferentiation. J Periodontol 83: 1028-1037, 2012.

2. Hota LS, Hanaway K, Hacker MR, Disciullo A, Elkadry E, Dramitinos P, Shapiro A, Ferzandi T and Rosenblatt PL: TVT-Secur (Hammock) versus TVT-Obturator: a randomized trial of suburethral sling operative procedures. Female Pelvic Med Reconstr Surg 18: 41-45, 2012.

3. Zhang W, Chancey AL, Tzeng HP, Zhou Z, Lavine KJ, Gao F, Sivasubramanian N, Barger PM and Mann DL: The development of myocardial fibrosis in transgenic mice with targeted overexpression of tumor necrosis factor requires mast cell-fibroblast interactions. Circulation 124: 2106-2116, 2011.

4. Khandwala S, Jayachandran C and Sengstock D: Experience with TVT-SECUR sling for stress urinary incontinence: a 141-case analysis. Int Urogynecol J Pelvic Floor Dysfunct 21: 767-772, 2010.

5. Uckert S, Oelke M, Waldkirch E, Stief CG, Albrecht K, Tröger HD, Jonas U, Andersson KE and Hedlund P: Cyclic adenosine monophosphate and cyclic guanosine monophosphate-phosphodiesterase isoenzymes in human vagina: relation to nitric oxide synthase isoforms and vasoactive intestinal polypeptide-containing nerves. Urology 65: 604-610, 2005. 
6. Skjeldestad FE and Hagen B: Long-term consequences of gynecological cancer treatment on urinary incontinence: a population-based cross-sectional study. Acta Obstet Gynecol Scand 87: 469-475, 2008.

7. Pickens RB, Klein FA, Mobley JD III and White WM: Single incision mid-urethral sling for treatment of female stress urinary incontinence. Urology 77: 321-324, 2011.

8. Yamamoto T, Gotoh M, Hattori R, Toriyama K, Kamei Y, Iwaguro H, Matsukawa Y and Funahashi Y: Periurethral injection of autologous adipose-derived stem cells for the treatment of stress urinary incontinence in patients undergoing radical prostatectomy: report of two initial cases. Int J Urol 17: 75-82, 2010.

9. Hwang JH, Kim IG, Piao S, Jung AR, Lee JY, Park KD and Lee JY: Combination therapy of human adipose-derived stem cells and basic fibroblast growth factor hydrogel in muscle regeneration. Biomaterials 34: 6037-6045, 2013.

10. Dal Moro F: The role of lysyl oxidase-like 1 and fibulin-5 in the development of atherosclerosis and pelvic organ prolapse. J Biomed Res 27: 242, 2013.

11. Thom DH, van den Eeden SK, Ragins AI, Wassel-Fyr C Vittinghof E, Subak LL and Brown JS: Differences in prevalence of urinary incontinence by race/ethnicity. J Urol 175 259-264, 2006.

12. Cabello-Verrugio C, Córdova G, Vial C, Zúñiga LM and Brandan E: Connective tissue growth factor induction by lysophosphatidic acid requires transactivation of transforming growth factor type $\beta$ receptors and the JNK pathway. Cell Signal 23: 449-457, 2011.
13. Copas P, Bukovsky A, Asbury B, Elder RF and Caudle MR: Estrogen, progesterone, and androgen receptor expression in levator ani muscle and fascia. J Womens Health Gend Based Med 10: 785-795, 2001.

14. Rajamaheswari N, Agarwal S,Chhikara AB and Seethalakshmi K Anterior bladder flap neo urethra as treatment for stress urinary incontinence due to developmental urogenital anomaly. Urol Ann 5: 215-217, 2013

15. Schreiner L, Santos TG, Souza AB, Nygaard CC and Silva Filho IG: Electrical stimulation for urinary incontinence in women: a systematic review. Int Braz J Urol 39: 454-464, 2013.

16. Wang S and Zhang S: Simultaneous perineal ultrasound and vaginal pressure measurement prove the action of electrical pudendal nerve stimulation in treating female stress incontinence. BJU Int 110: 1338-1343, 2012.

17. Berman JR, Adhikari SP and Goldstein I: Anatomy and physiology of female sexual function and dysfunction: classification, evaluation and treatment options. Eur Urol 38: 20-29, 2000

18. Oh SJ, Hong SK, Kim SW and Paick JS: Histological and functional aspects of different regions of the rabbit vagina. Int J Impot Res 15: 142-150, 2003.

19. Mammoto $\mathrm{T}$ and Ingber DE: Mechanical control of tissue and organ development. Development 137: 1407-1420, 2010.

20. Miyata A, Shioda S and Baba A: The 9th International Symposium on VIP, PACAP, and related peptides. J Mol Neurosci 42: 264-265, 2010. 